\title{
Role of the long form leptin receptor and of the STAT3 signaling pathway in colorectal cancer progression
}

\author{
TAKASHI UCHIYAMA ${ }^{1}$, HIROKAZU TAKAHASHI ${ }^{1}$, HIROKI ENDO ${ }^{1}$, MICHIKO SUGIYAMA $^{1}$, \\ EIJI SAKAI ${ }^{1}$, KUNIHIRO HOSONO $^{1}$, YOJI NAGASHIMA ${ }^{2}$, YOSHIAKI INAYAMA ${ }^{2}$, \\ KOICHIRO WADA ${ }^{3}$, YOSHITAKA HIPPO ${ }^{4}$ and ATSUSHI NAKAJIMA ${ }^{1}$ \\ ${ }^{1}$ Divisions of Gastroenterology, ${ }^{2}$ Pathology, Yokohama City University School of Medicine, Kanazawa-ku, Yokohama; \\ ${ }^{3}$ Department of Pharmacology, Graduate School of Dentistry, Osaka University, Suita, Osaka; ${ }^{4}$ Division of \\ Cancer Development System, National Cancer Center Research Institute, Chuo-ku, Tokyo, Japan
}

Received April 1,2011; Accepted May 23, 2011

DOI: $10.3892 /$ ijo.2011.1105

\begin{abstract}
Although a number of recent studies have reported the involvement of leptin in colorectal carcinogenesis, findings are contradictory and difficult to interpret. Our group has previously reported that leptin signaling might have an important role in the development of colorectal adenomas. In this study, we investigated leptin signaling in colorectal carcinogenesis focusing in particular on the differences in leptin signaling between colorectal adenoma and cancer. Whereas no significant differences in the serum leptin levels were observed among normal control subjects and adenoma/cancer patients, increased expression and activation of the long form leptin receptor (ObRL) was observed in colorectal adenoma and cancer tissues compared with the normal colorectal tissues. However, no significant differences were observed between the colorectal adenoma and cancer tissues. Significant increases in the phosphorylation levels of important molecules of the JAK/STAT signaling pathway, located downstream of leptin signaling, and transcriptional regulation of STAT3downstream target molecules were observed in colorectal adenoma tissue compared with the findings in normal colorectal tissues. Furthermore, these changes were significantly more pronounced in colorectal cancer compared to colorectal adenoma tissues. This is the first analysis of leptin and JAK/ STAT signaling in a human colorectal adenoma-carcinoma sequence. These results suggest that the STAT3-mediated leptin signaling through the activation of ObRL may be involved in colorectal carcinogenesis, both in adenoma formation and in the progression to cancer. STAT3 signaling in colorectal cancer may be mediated not only by leptin but by other factors.
\end{abstract}

Correspondence to: Dr Atsushi Nakajima, Division of Gastroenterology, Yokohama City University School of Medicine, 3-9 Fukuura, Kanazawa-ku, Yokohama, Kanagawa 236-0004, Japan E-mail: nakajima-tky@umin.ac.jp

Key words: leptin, colorectal cancer, leptin receptor, STAT3

\section{Introduction}

A number of recent studies have reported that leptin is involved in colorectal carcinogenesis (1-3). Although several studies have demonstrated the role of leptin as an important growth factor for colorectal cancer cell lines in vitro (1-3), the relationship between leptin signaling and colorectal cancer development in vivo still remains controversial. Animal experiments conducted in vivo in rodent models of colorectal carcinogenesis have revealed no effects of leptin signaling on colorectal carcinogenesis $(2,4,5)$. In contrast, many previous clinical studies have shown a significant association between serum leptin levels and the presence of colorectal cancer (6-13). Thus, the results of previous clinical studies and animal experiments are contradictory and difficult to interpret.

In a previous study, we demonstrated an important role of leptin signaling in the development of colorectal adenoma (14). Although we found no association between serum leptin levels and the presence of colorectal adenoma, increased expression of the long form leptin receptor (ObRL) and activation of JAK/ STAT signaling were observed in colorectal adenoma tissues as compared with the observations in normal tissues. These results clearly suggest that the leptin signaling pathway plays a crucial role in the growth of colorectal adenoma.

Meanwhile, the progression of adenoma to cancer is another important step in colorectal carcinogenesis. However, the role of leptin signaling in the progression of adenoma to cancer is still unclear. Therefore, in this study, we investigated the leptin signaling in colorectal carcinogenesis especially focused on the differences in leptin signaling between colorectal adenoma and cancer.

\section{Materials and methods}

Study population. The study protocol was approved by the Yokohama City University Hospital Ethics Committee. Written informed consent was obtained from all the subjects prior to their participation in the study.

Thirty-one patients diagnosed as having colorectal cancer between January 2008 and July 2009 at Yokohama City 
Table I. Characteristics of the study patients.

\begin{tabular}{lcccc}
\hline Characteristics & Controls & Adenoma & Cancer & P-value \\
\hline No. of patients & 68 & 60 & 31 & 0.53 \\
Age (year) & $62.0 \pm 14.0$ & $64.3 \pm 11.1$ & $64.3 \pm 8.7$ & 0.37 \\
Gender, male/female & $37 / 31$ & $40 / 20$ & $19 / 12$ & 0.52 \\
Waist circumference $(\mathrm{cm})$ & $84.3 \pm 10.1$ & $85.5 \pm 8.3$ & $84.0 \pm 9.6$ & 0.10 \\
BMI $\left(\mathrm{kg} / \mathrm{m}^{2}\right)$ & $22.6 \pm 3.5$ & $23.5 \pm 2.7$ & $22.4 \pm 3.2$ & 0.08 \\
VFA $\left(\mathrm{cm}^{2}\right)$ & $74.6 \pm 49.7$ & $91.0 \pm 39.0$ & $83.7 \pm 49.1$ & 0.42 \\
FBS $(\mathrm{mg} / \mathrm{dl})$ & $111.7 \pm 27.2$ & $105.0 \pm 25.5$ & $103.9 \pm 18.8$ & 0.99 \\
HbA1c $(\%)$ & $5.7 \pm 1.2$ & $5.5 \pm 2.9$ & $5.4 \pm 0.6$ & 0.69 \\
Leptin $(\mathrm{ng} / \mathrm{ml})$ & $5.4 \pm 4.2$ & $5.4 \pm 4.0$ & $4.9 \pm 4.3$ & \\
\hline
\end{tabular}

Data are shown as the mean \pm standard deviation (SD). BMI, body mass index; VFA, visceral fat area; FBS, fasting blood sugar. Statistical analysis was performed by the Kruskal-Wallis test.

University Hospital, 68 control subjects in whom colonoscopy confirmed the absence of any colorectal polyps, and 60 adenoma patients were recruited for this study. The exclusion criteria were subjects with familial adenomatous polyposis, inflammatory bowel disease, radiation colitis or any malignant disease, and subjects with a previous history of colectomy, gastrectomy or colorectal polypectomy. We also excluded colorectal cancer patients with weight loss of over $5 \%$ in the previous 6 months, to avoid the confounding effects of the effects of weight loss on the serum leptin levels.

Collection and analysis of blood samples for determination of the leptin levels. Blood samples were obtained in the morning on the day of colonoscopy, after the subjects had fasted overnight. Serum leptin levels were measured with an enzyme-linked immunosorbent assay of human leptin (SRL Co., Tokyo, Japan).

Immunohistochemical analyses. The expressions of $\mathrm{ObR}$ and phospho-STAT3 (p-STAT3) were investigated in normal colorectal, adenoma and cancer tissues. A total 40 samples of normal colorectal and adenoma tissues, and 20 samples of cancer tissues were obtained from the study subjects. The tissue samples were isolated, formalin-fixed and paraffin-embedded; the paraffin-embedded samples were then deparaffinized and rehydrated. Sections prepared from the specimens were incubated with antibodies for ObR (1:50, Santa Cruz Biotechnology, Santa Cruz, CA, USA) and p-STAT3 (Tyr 705) (1:50, Cell Signaling Technology, Danvers, MA, USA) as the primary antibodies, using an LSAB2 kit (Dako Cytomation, Glostrup, Denmark). They were then incubated with biotinylated immunoglobulin as the secondary antibody and treated with peroxidase-conjugated streptavidin. The antibody complex was visualized with 3,3'-diaminobenzidine, tetrahydrochloride (Dojindo Laboratories, Kumamoto, Japan). The expressions of ObR and p-STAT3 were analyzed with light microscopy in 10 different fields of each section, and the mean percentage of the gland cells that showed positive staining was scored by two pathologists. The ObR and p-STAT3 expressions were classified into two categories depending on the percentage of cells showing positive staining: negative,
$0-15 \%$ of all the gland cells showing positive staining; positive, $>15 \%$ of all gland cells showing positive staining, as previously described (15).

Western blot analysis. Samples of normal colorectal tissue and colorectal adenoma and cancer tissues were isolated, and the extracted protein was separated with sodium dodecylsulfate polyacrylamide gel electrophoresis (SDS-PAGE); the separated proteins were then transferred to a polyvinylidene difluoride (PVDF) membrane (Amersham, London, UK) and probed with primary antibodies specific for phospho-ObR (p-ObR) (Tyr 1141), p-ObR (Tyr 985), ObR (Santa Cruz Biotechnology), phospho-JAK2 (p-JAK2), JAK2, p-STAT3 (Tyr 705) STAT3 (Cell Signaling Technology), and glyceraldehyde-3-phosphate dehydrogenase (GAPDH) (Trevigen, Gaithersburg, MD, USA). Horseradish peroxidase-conjugated secondary antibodies and the ECL detection kit (Amersham) were used for the detection of specific proteins.

Real-time RT-PCR. Samples of normal colorectal tissues and colorectal adenoma and cancer tissues were isolated, and total-RNA from the colorectal cancer and normal colorectal tissues was extracted using the RNeasy mini kit (Qiagen, Hilden, Germany). For the real-time reverse-transcriptase polymerase chain reaction, total-RNA was reverse-transcribed into cDNA and amplified with the real-time quantitative polymerase chain reaction method using the StepOne Plus Real-Time PCR System (Applied Biosystems, Foster City, CA, USA). Probes and primer pairs specific for ObRL, short form leptin receptor (ObRS), BclX, c-Myc, cyclin D1, cdc2, cyclin B1, VEGF and 18S were purchased from Applied Biosystems. The concentrations of the target genes were determined using the competitive computed tomography method and the values were normalized to the internal control.

Statistical analysis. Statistical analyses were performed using the Kruskal-Wallis test and the Fisher's PLSD test. All analyses were performed using the StatView software (SAS Institute, Cary, NC, USA). A P-value $<0.05$ was regarded as denoting statistical significance. 


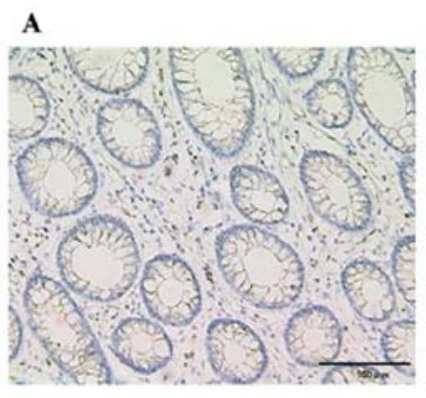

D
B

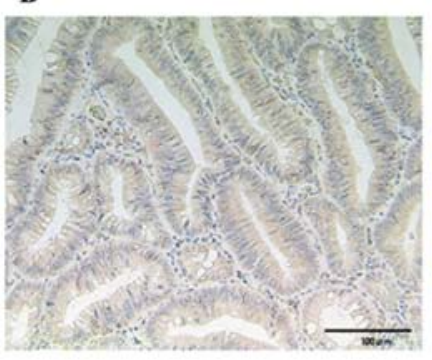

C

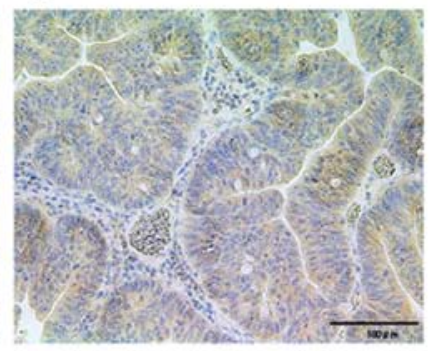

$\mathbf{E}$
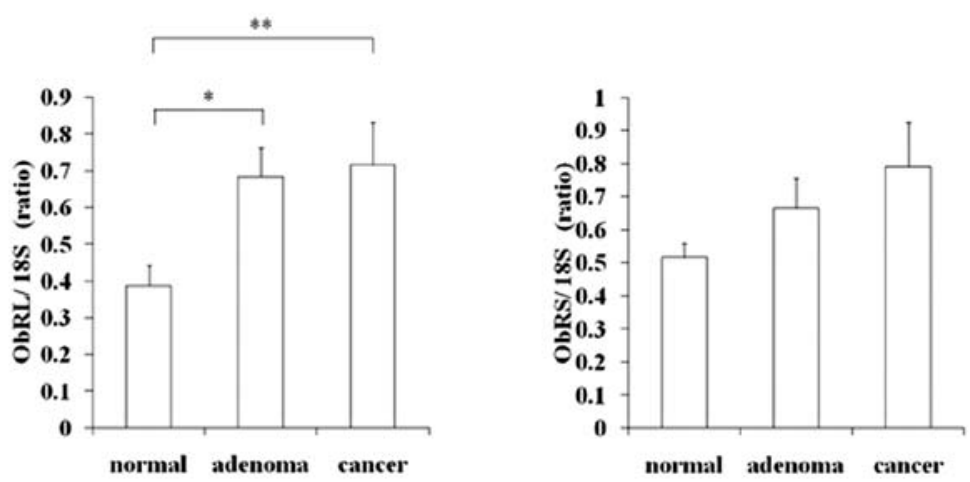

Figure 1. (A-C) Immunohistochemical staining for leptin receptor (ObR) and (D and E) mRNA levels of the long and short forms of ObR, namely, ObRL and ObRS. (A) Normal colorectal tissue, (B) colorectal adenoma tissue and (C) colorectal cancer tissue. Scale bar, $100 \mu \mathrm{m}$. The relative mRNA expressions of (D) ObRL and (E) ObRS in normal colorectal, and colorectal adenoma and cancer tissues are expressed as the ratios relative to the expression level of 18S. Each column represents the mean $\pm \mathrm{SEM} .{ }^{*} \mathrm{P}<0.05 ;{ }^{* * *} \mathrm{P}<0.01$

\section{Results}

Serum leptin levels and colorectal cancer. The clinical characteristics of the colorectal cancer patients and the control subjects are shown in Table I. No significant differences in the serum leptin levels were observed in these groups. There were also no significant differences in the age, BMI or other obesity-related factors in these groups.

Leptin receptor $(\mathrm{ObR})$ expression in normal colorectal, colorectal cancer and adenoma tissues. To examine the expression levels of the ObR in normal colorectal, colorectal adenoma and cancer tissues, immunohistochemical staining and gene expression analyses were performed. Strong expression of ObR was found in the colorectal adenoma and cancer tissues, but not in the gland cells of normal colorectal tissue (Fig. 1A-C). The detection frequency of ObR in colorectal cancer was $90.0 \%$ (18/20) and that in colorectal adenoma was $67.5 \%$ (27/40). In addition, the mRNA expression levels of ObRL and the ObRS in the normal colorectal and colorectal cancer and adenoma tissues were investigated. The expression levels of ObRL mRNA were significantly higher in the colorectal adenoma and cancer tissues than in the normal colorectal tissue (Fig. 1D). No significant differences in the expression levels were observed between colorectal cancer and adenoma tissues. The expression levels of ObRS were also higher, but not significantly, in the colorectal adenoma and cancer tissues than in the normal colorectal tissue (Fig. 1E).

Activation of leptin receptor. We also investigated the activation of the leptin receptor with Western blot analysis of the phosphorylation of the cytoplasmic domain of ObRL. A significant increase in ObR expression and phosphorylation of ObRL-Tyr1141 was observed in the samples collected from the colorectal adenoma and cancer tissues as compared with the findings in normal colorectal tissue (Fig. 2A and B). No significant differences were observed between the colorectal cancer and colorectal adenoma tissues. As the phosphorylation of Tyr 1141 in ObRL is required for leptin-induced activation of STAT3 (16), these results indicate that overexpression and activation of ObR are induced in colorectal adenoma and cancer tissues. In contrast, no significant difference was observed in the phosphorylation level of Tyr 985 in ObR, which is required for activation of the ERK (extracellular-signal-regulated kinase) signaling pathway (Fig. 2C) (17).

Phosphorylated STAT3 in colorectal cancer. To confirm the activation of the STAT3 pathway, immunohistochemical staining and Western blot analysis for p-STAT3 were performed. Increased expression of p-STAT3 was observed in the nuclei of gland cells of the colorectal adenoma and cancer tissue specimens, indicating that STAT3 was activated in these tissues. On the other hand, no expression was observed in normal colorectal tissue specimens (Fig. 3). The detection frequency of p-STAT3 in colorectal cancer was 85\% (17/20) and that in adenoma was $57.5 \%$ (23/40). Western blot analysis demonstrated that the levels of p-JAK2 and p-STAT3 were significantly higher in colorectal cancer tissue than in the normal colorectal and adenoma tissues (Fig. 4). These results suggest that activation of STAT3 was significantly increased in colorectal adenoma and cancer tissues compared with normal colorectal tissues, with significantly increased activation also 
A

ObR

GAP
B

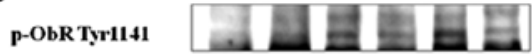
GAP

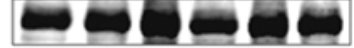

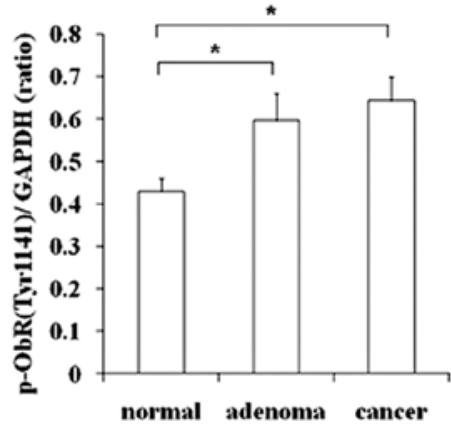

C

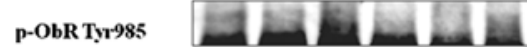
GAP

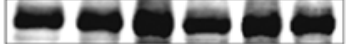

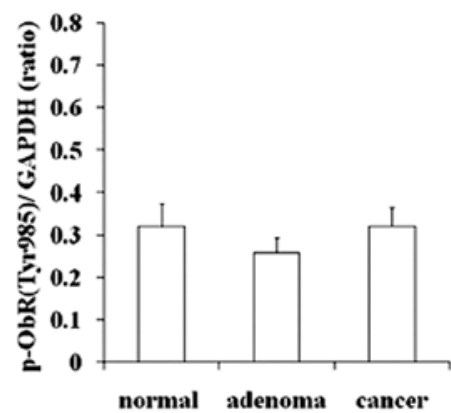

Figure 2. Western blot analysis for ObR and phosphorylated ObR (Tyr 1141 or Tyr 985). (A) ObR, (B) Tyr 1141-phosphorylated and (C) Tyr 985-phosphorylated ObR. Upper panels: representative Western blot analyses for ObR, Tyr 1141-phosphorylated and Tyr 985-phosphorylated ObR. Lanes 1 and 2: normal colorectal tissues; lanes 3 and 4: adenoma tissues; lanes 5 and 6: cancer tissues. Lower panels: ratios of the expression levels of ObR, Tyr 1141-phosphorylated and Tyr 985-phosphorylated ObR to the expression level of GAPDH are shown. Each column represents the mean \pm SEM. "P<0.05.

A

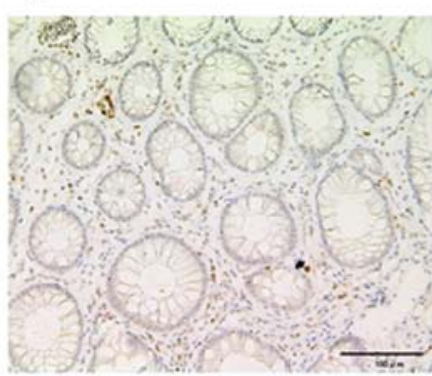

B

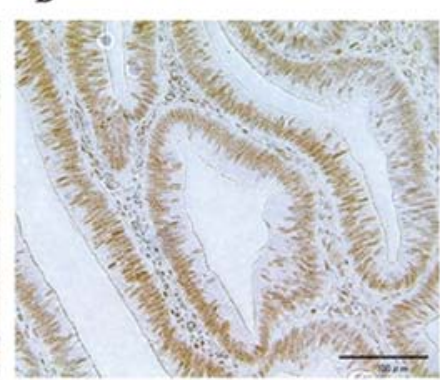

C

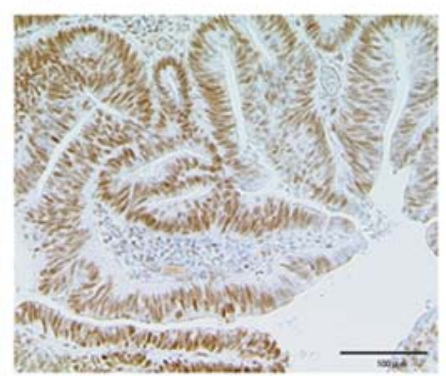

Figure 3. Immunohistochemical staining for phosphorylated STAT3. (A) Normal colorectal tissue, (B) colorectal adenoma tissue and (C) colorectal cancer tissue. Scale bar, $100 \mu \mathrm{m}$.

A

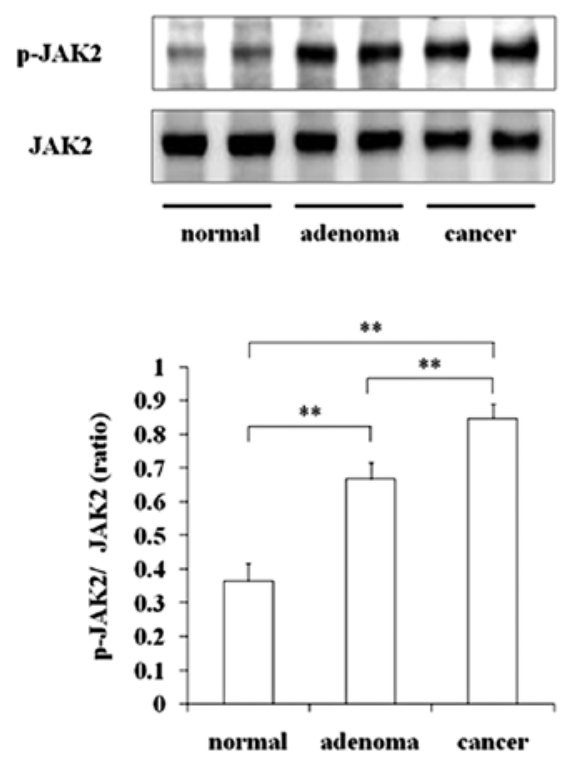

B

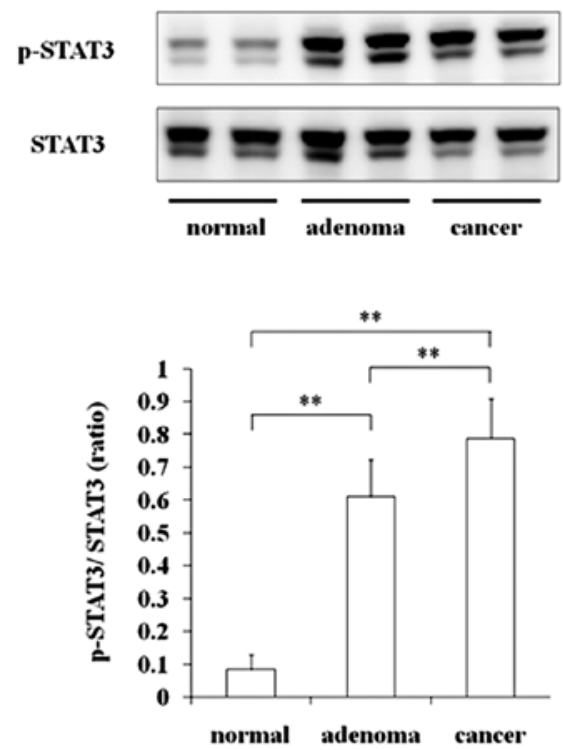

Figure 4. Western blot analysis for phosphorylated JAK2 and STAT3. (A) Phosphorylated JAK2 and (B) phosphorylated STAT3. Upper panels: Representative Western blot analyses for phosphorylated and total levels of JAK2 and STAT3. Lanes 1 and 2: normal colorectal tissue; lanes 3 and 4: adenoma tissue; lanes 5 and 6: cancer tissue. Lower panels: ratios of the levels of the phosphorylated proteins to the total protein level. Each column represents the mean $\pm \mathrm{SEM}$. ${ }^{* *} \mathrm{P}<0.01$. 
A

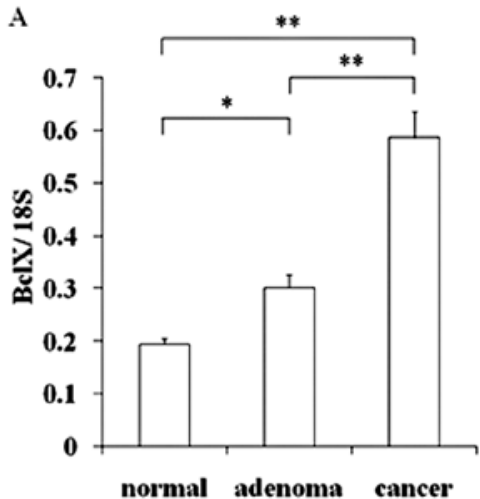

D

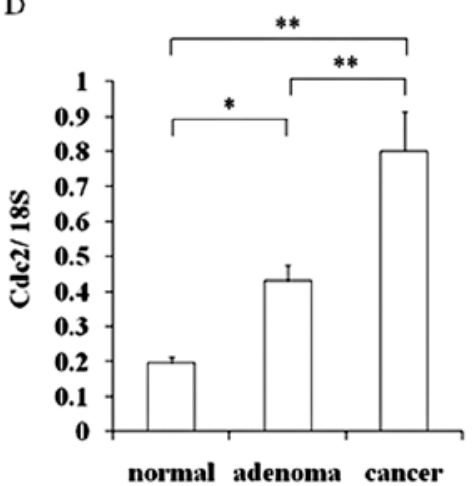

B

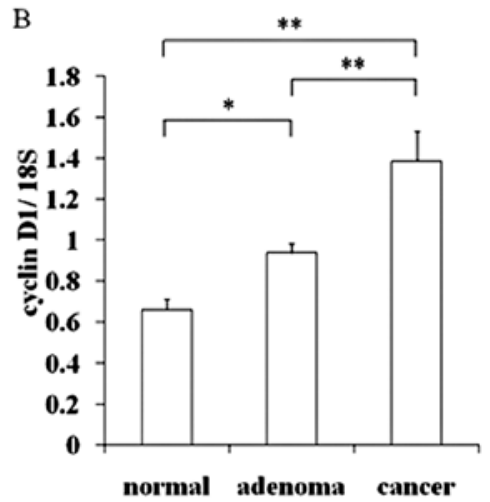

E

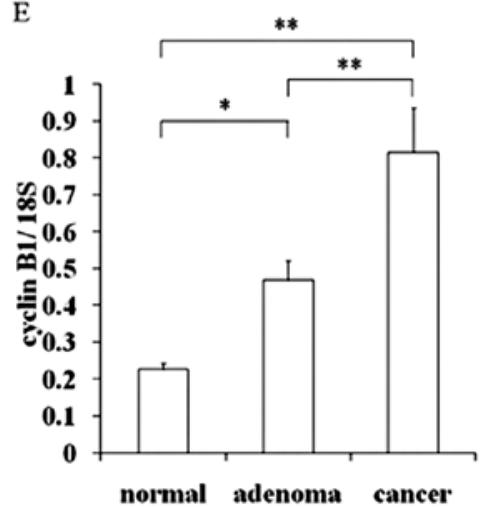

C

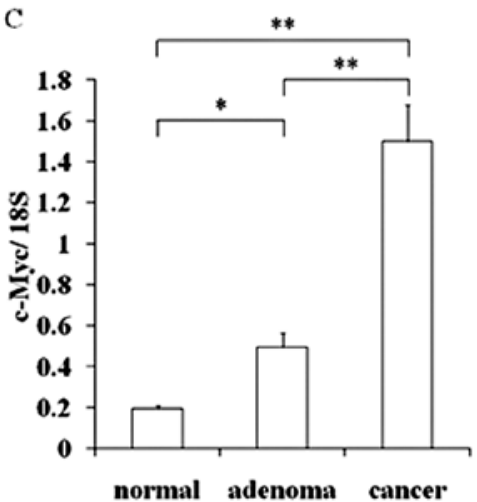

F

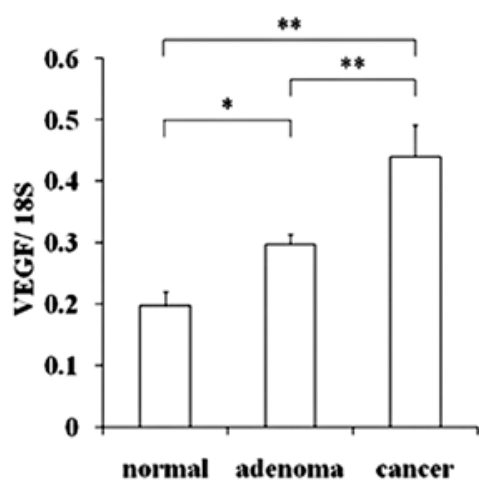

Figure 5. Expressions of downstream genes encoded by STAT3 transcriptional regulation in normal colorectal, colorectal cancer and adenoma tissues. The relative mRNA expressions of (A) BclX, (B) cyclin D1, (C) c-Myc, (D) cdc2, (E) cyclin B1 and (F) VEGF expressions in normal colorectal, adenoma and cancer tissues were expressed as ratios relative to the expression level of $18 \mathrm{~S}$. Each column represents the mean $\pm \mathrm{SEM}$. ${ }^{*} \mathrm{P}<0.05 ;{ }^{* * *} \mathrm{P}<0.01$.

seen in colorectal cancer tissues compared with colorectal adenoma specimens.

STAT3-mediated transcriptional regulation of gene expression. Alterations of the mRNA levels of the genes encoded by STAT3 were investigated with real-time RT-PCR. The expression levels of the apoptosis-suppressing protein $\mathrm{BclX}$, the late $\mathrm{G} 1$ to $\mathrm{G} 1 / \mathrm{S}$ phase proteins cyclin D1 and c-Myc, the $\mathrm{G} 2 / \mathrm{M}$ phase proteins cdc2 and cyclin B1 (18), and of the genes encoding the angiogenesis protein VEGF (19) were significantly higher in colorectal cancer tissue than in the normal colorectal and colorectal adenoma tissues (Fig. 5). These results suggest strong activation of the JAK/STAT signaling pathway in colorectal cancer tissues as compared with normal colorectal and colorectal adenoma tissues.

\section{Discussion}

Previous studies have suggested the existence of an association between the serum leptin levels and the development of colorectal cancer (6-13). In this study, however, we observed no statistically significant differences in the serum leptin levels among the normal control and colorectal adenoma/ cancer patients. Stachowicz et al reported that the serum leptin levels were not dependent on the clinical or pathological stage of progression of colorectal cancer (20). Combined with this report, the results of our present study suggest that the serum leptin levels are not associated with the presence of tumor in the course of colorectal carcinogenesis.
We observed significantly higher expression and activation levels of ObRL in colorectal adenoma and cancer tissues than in normal colorectal tissue. In addition we focused on the differences in the expression and activation levels between colorectal adenoma and cancer tissues, and observed no significant differences in the expression or activation levels of ObRL between colorectal adenoma and cancer tissues. These results suggest that leptin may play a crucial role not only in the development of colorectal adenoma but equally in that of colorectal cancer.

We also showed activation of the JAK/STAT pathway which is located downstream of the activation of leptin receptor signaling. Several other studies have also reported more pronounced activation of JAK/STAT signaling in colorectal cancer tissues than in normal colorectal tissue (15,21-23). These reports lend support to our present observations. However, our results showed a discrepancy between the expression and activation levels of $\mathrm{ObR}$ and the activation of JAK/STAT signaling. Although we observed no significant differences in the expression or activation levels of ObR between colon cancer and adenoma tissues, activation of the JAK/STAT pathway was significantly more pronounced in colorectal cancer tissue than in colorectal adenoma tissue. JAK/STAT signaling has been reported to be mediated not only by leptin receptor signaling, but also by several cytokines and hormones, such as IL-6 (24-26). Therefore, based on our findings we suggested that STAT3 was phosphorylated by leptin signaling in both colorectal cancer and adenoma tissues. In addition, in colorectal cancer, we speculated that STAT3 may also be phosphorylated by other signaling processes. 
These results suggest that leptin signaling in the activation of STAT3 was important especially in colorectal adenoma that was in an early phase of colorectal carcinogenesis.

In conclusion, no significant differences in the serum leptin levels were observed among normal control subjects and adenoma/cancer patients. We observed increased expression and activation of the ObRL in colorectal cancer and adenoma tissues compared with normal colorectal tissue. Significantly increased phosphorylation levels of the important molecules of the JAK/STAT signaling pathway and transcriptional regulation of the STAT3-downstream target molecules were observed in colorectal adenoma tissues as compared with the findings in normal colorectal tissue. Furthermore, these changes were more pronounced in colorectal cancer tissue than in colorectal adenoma tissue. Further investigations are required to clarify the exact roles of leptin signaling and JAK/ STAT signaling in colorectal carcinogenesis, and the detailed mechanisms underlying colorectal carcinogenesis.

\section{Acknowledgements}

We thank Ms. Machiko Hiraga for her technical assistance. This study was supported in part by a Grant-in-Aid for research on the Third-Term Comprehensive Control Research for Cancer from the Ministry of Health, Labour and Welfare, Japan, to A.N., a grant from the National Institute of Biomedical Innovation (NBIO) to A.N., a grant from the Ministry of Education, Culture, Sports, Science and Technology, Japan (KIBAN-B) to A.N., and the grant program, 'Collaborative Development of Innovative Seed' from the Japan Science and Technology Agency (JST).

\section{References}

1. Hardwick JC, Van Den Brink GR, Offerhaus GJ, Van Deventer SJ and Peppelenbosch MP: Leptin is a growth factor for colonic epithelial cells. Gastroenterology 121: 79-90, 2001.

2. Aparicio T, Kotelevets L, Tsocas A, Laigneau JP, Sobhani I, Chastre E and Lehy T: Leptin stimulates the proliferation of human colon cancer cells in vitro but does not promote the growth of colon cancer xenografts in nude mice or intestinal tumorigenesis in Apc(Min/+) mice. Gut 54: 1136-1145, 2005.

3. Ogunwobi OO and Beales IL: The anti-apoptotic and growth stimulatory actions of leptin in human colon cancer cells involves activation of JNK mitogen activated protein kinase, JAK2 and PI3 kinase/Akt. Int J Colorectal Dis 22: 401-409, 2007.

4. Aparicio T, Guilmeau S, Goiot H, Tsocas A, Laigneau JP, Bado A, Sobhani I and Lehy T: Leptin reduces the development of the initial precancerous lesions induced by azoxymethane in the rat colonic mucosa. Gastroenterology 126: 499-510, 2004.

5. Hirose Y, Hata K, Kuno T, Yoshida K, Sakata K, Yamada Y, Tanaka T, Reddy BS and Mori H: Enhancement of development of azoxymethane-induced colonic premalignant lesions in C57BL/KsJ-db/db mice. Carcinogenesis 25: 821-825, 2004.

6. Kumor A, Daniel P, Pietruczuk M and Małecka-Panas E: Serum leptin, adiponectin, and resistin concentration in colorectal adenoma and carcinoma (CC) patients. Int J Colorectal Dis 24: 275-281, 2009.

7. Arpaci F, Yilmaz MI, Ozet A, Ayta H, Ozturk B, Komurcu S and Ozata M: Low serum leptin level in colon cancer patients without significant weight loss. Tumori 88: 147-149, 2002.

8. Bolukbas FF, Kilic H, Bolukbas C, Gumus M, Horoz M, Turhal NS and Kavakli B: Serum leptin concentration and advanced gastrointestinal cancers: a case controlled study. BMC Cancer 24 29,2004 .
9. Wallace AM, Sattar N and McMillan DC: Effect of weight loss and the inflammatory response on leptin concentrations in gastrointestinal cancer patients. Clin Cancer Res 4: 2977-2979, 1998.

10. Tessitore L, Vizio B, Jenkins O, De Stefano I, Ritossa C, Argiles JM, Benedetto $\mathrm{C}$ and Mussa A: Leptin expression in colorectal and breast cancer patients. Int J Mol Med 5: 421-426, 2000.

11. Stattin P, Palmqvist R, Söderberg S, Biessy C, Ardnor B, Hallmans G, Kaaks R and Olsson T: Plasma leptin and colorectal cancer risk: a prospective study in Northern Sweden. Oncol Rep 10: 2015-2021, 2003.

12. Stattin P, Palmqvist R, Söderberg S, Biessy C, Ardnor B, Hallmans G, Kaaks R and Olsson T: Obesity and colon cancer: does leptin provide a link? Int J Cancer 109: 149-152, 2004.

13. Tamakoshi K, Toyoshima H, Wakai K, Kojima M, Suzuki K, Watanabe Y, Hayakawa N, Yatsuya H, Kondo T, Tokudome S, Hashimoto S, Suzuki S, Kawado M, Ozasa K, Ito Y and Tamakoshi A: Leptin is associated with an increased female colorectal cancer risk: a nested case-control study in Japan. Oncology 68: 454-461,2005.

14. Uchiyama T, Takahashi H, Sugiyama M, Sakai E, Endo H, Hosono K, Yoneda K, Yoneda M, Inamori M, Nagashima Y, Inayama Y, Wada $\mathrm{K}$ and Nakajima A: Leptin receptor is involved in STAT3 activation in human colorectal adenoma. Cancer Sci 102: 367-372, 2011.

15. Kusaba T, Nakayama T, Yamazumi K, Yakata Y, Yoshizaki A, Nagayasu T and Sekine I: Expression of p-STAT3 in human colorectal adenocarcinoma and adenoma; correlation with clinicopathological factors. J Clin Pathol 58: 833-838, 2005.

16. Cao Q, Mak KM, Ren C and Lieber CS: Leptin stimulates tissue inhibitor of metalloproteinase-1 in human hepatic stellate cells: respective roles of the JAK/STAT and JAK-mediated $\mathrm{H}_{2} \mathrm{O}_{2-}$ dependant MAPK pathways. J Biol Chem 279: 4292-4304, 2004.

17. Burguera B, Couce ME, Long J, Lamsam J, Laakso K, Jensen MD, Parisi JE and Lloyd RV: The long form of the leptin receptor (OB-Rb) is widely expressed in the human brain. Neuroendocrinology 71 : 187-195, 2000.

18. Bollrath J, Phesse TJ, von Burstin VA, Putoczki T, Bennecke M, Bateman T, Nebelsiek T, Lundgren-May T, Canli O, Schwitalla S, Matthews V, Schmid RM, Kirchner T, Arkan MC, Ernst M and Greten FR: gp130-mediated STAT3 activation in enterocytes regulates cell survival and cell-cycle progression during colitisassociated tumorigenesis. Cancer Cell 15: 91-102, 2009.

19. Niu G, Wright KL, Huang M, Song L, Haura E, Turkson J, Zhang S, Wang T, Sinibaldi D, Coppola D, Heller R, Ellis LM, Karras J, Bromberg J, Pardoll D, Jove R and Yu H: Constitutive STAT3 activity up-regulates VEGF expression and tumor angiogenesis. Oncogene 21: 2000-2008, 2002.

20. Stachowicz M, Mazurek U, Nowakowska-Zajdel E, Niedworok E, Fatyga $\mathrm{E}$ and Muc-Wierzgon M: Leptin and its receptors in obese patients with colorectal cancer. J Biol Regul Homeost Agents 24: 287-295, 2010.

21. Park JK, Hong R, Kim KJ, Lee TB and Lim SC: Significance of p-STAT3 expression in human colorectal adenocarcinoma. Oncol Rep 20: 597-604, 2008.

22. Ma XT, Wang S, Ye YJ, Du RY, Cui ZR and Somsouk M: Constitutive activation of Stat 3 signaling pathway in human colorectal carcinoma. World J Gastroenterol 10: 1569-1573, 2004.

23. Corvinus FM, Orth C, Moriggl R, Tsareva SA, Wagner S, Pfitzner EB, Baus D, Kaufmann R, Huber LA, Zatloukal K, Beug H, Ohlschläger P, Schütz A, Halbhuber KJ and Friedrich K: Persistent STAT3 activation in colon cancer is associated with enhanced cell proliferation and tumor growth. Neoplasia 7: 545-555, 2005.

24. Aggarwal BB, Kunnumakkara AB, Harikumar KB, Gupta SR, Tharakan ST, Koca C, Dey S and Sung B: Signal transducer and activator of transcription-3, inflammation, and cancer: how intimate is the relationship? Ann NY Acad Sci 1171: 59-76, 2009.

25. Murray PJ: The JAK/STAT signaling pathway: input and output integration. J Immunol 178: 2623-2629, 2007.

26. Zhong Z, Wen Z and Darnell JE Jr: Stat3: a STAT family member activated by tyrosine phosphorylation in response to epidermal growth factor and interleukin-6. Science 264: 95-98, 1994. 